\title{
SpIOMM and SITELLE: Wide-field Imaging FTS for the Study of Galaxy Evolution
}

\section{Laurent Drissen ${ }^{1}$, Anne-Pier Bernier ${ }^{1}$, Carmelle Robert ${ }^{1}$, and the SpIOMM \& SITELLE team}

${ }^{1}$ Département de physique, de génie physique et d'optique, Université Laval, Québec, Canada email: ldrissen@phy.ulaval.ca

\begin{abstract}
SpIOMM, a wide-field Imaging Fourier Transform Spectrometer attached to the Mont Mégantic 1.6-m telescope, is capable of obtaining the visible spectrum of every source of light in a 12 arcminute field of view, with a spectral resolution ranging from $\mathrm{R}=1$ (wide-band image) to $\mathrm{R}=25000$, resulting in 1.7 million spectra with a spatial resolution of one arcsecond. SITELLE will be a similar instrument attached to the Canada-France-Hawaii telescope, and will be in operation in early 2013. We present a short description of these instruments and illustrate their capabilities to study nearby galaxies with the results of a data cube of M51.
\end{abstract}

Keywords. instrumentation: spectrographs, galaxies: abundances, galaxies: individual (M51)

\section{Introduction}

Major breakthroughs have also been obtained in the study of galaxies over the last decade by combining imagery and spectroscopy into a single experimental observation technique that produces cubes of data, Integral Field Units (IFUs). Given the limitations of current array detectors however, different instrument concepts convey different trades that each enhances the possibility of discovery for a given science program category. The ability to cover a greater area than the classical spectrometer slit has been the driving motivation behind these 3-D instrument developments.

For example, SAURON (Bacon et al. 2001; Emsellem, these proceedings) has revolutionized the study of late-type galaxies by providing a relatively large field field of view $(41 \times 33$ arcseconds) at a spectral resolution $\mathrm{R} \sim 1000$. A similar, but huge and much more complex instrument, MUSE, is currently being built for the VLT (Bacon et al. 2010); it will cover a $1 \times 1$ arcminute field of view with a resolution $R \sim 1000-4000$. Another wide-field IFU, VIRUS-P, is being used at the McDonald Observatory's 2.7-m Harlan J. Smith telescope; it samples a $1.7^{\prime} \times 1.7^{\prime}$ field of view with a 0.3 filling factor (Blanc et al. 2009). The field of view and total throughput of dispersive IFUs are however limited.

A large number of research programs would benefit from an instrument capable of simultaneously obtaining spatially resolved, high quality spectra on extended areas (of the order of 10 arcminutes) and with a resolution up to $\mathrm{R} \sim 10^{4}$. Imaging Fourier transform spectroscopy is very promising in that regard. Based on the principle of the Michelson interferometer, Fourier Transform Spectrometers (FTS) are extremely efficient because all photons are collected and analyzed. We have designed, built and used a wide field imaging FTS, SpIOMM, for the Observatoire du Mont Mégantic's 1.6-m telescope and are now working on a similar instrument, SITELLE, for the Canada-France-Hawaii telescope (CFHT). 


\section{Imaging FTS}

While FTS are widely used in remote sensing, atmospheric analysis and chemical applications, as well as planetary exploration (Flasar et al. 2004), the use of FTS is not widespread in astronomy, mostly because of the technical difficulties in building such instruments. Let's however mention the BEAR imaging FTS, which has been used at the CFHT (Paumard et al. 2004), and the more recent imaging FTS mode of the SPIRE instrument onboard the Herschel space telescope (Griffin et al. 2010, Panuzzo et al. 2010).

With an imaging FTS, spectral information is obtained by acquiring multiple panchromatic images of the selected scene that are modulated in intensity in a precisely controlled fashion. The core of the instrument is a Michelson interferometer inserted in series within the camera optical design, which modulates the image intensity from total dark to total brightness depending on the optical path difference (OPD) between the two interferometer arms. This OPD being a function of wavelength, the resulting image intensity is the sum of all individual wavelength contributions. A recording of these panchromatic images generated at equally spaced OPD positions creates the raw data cube, thus producing a discrete interferogram for each pixel of the image from which spectral information can then be extracted. The spectrum of the source, at each pixel, is obtained from the Fourier transform of these individual interferograms. The net results are thus a deep panchromatic image of the target and a complete set of spectra, one for every pixel.

From our point of view, the niche of an astronomical IFTS clearly sits in the wide field coverage, moderate spectral resolution $(\mathrm{R} \sim 2000-10000)$ and the large waveband coverage, although it is interesting to note that the FTS was classically known for its ability to obtain very high spectral resolution of the targets. Two important advantages of an imaging FTS are its intrinsically high throughput and the fact that, contrary to conventional IFUs, there is no image reconstruction necessary. Review articles on the use of imaging FTS in astronomy can be found in Drissen et al. (2011) and Maillard et al. (2011).

\section{SpIOMM}

In order to demonstrate the capabilities of a wide-field imaging FTS working in the visible band, our group has designed and built SpIOMM at Université Laval in close collaboration with ABB-Bomem, a Québec City-based company.

SpIOMM is capable of obtaining spectra in selected bands of the visible spectrum (from 350 to $850 \mathrm{~nm}$ ) of every light source in a 12 arcminute (circular) field of view of the OMM 1.6-m telescope. Spectral resolution is variable, depending on the need of the observer, from $\mathrm{R}=1$ (broad-band image) to $\mathrm{R}=25000$. We however use SpIOMM in selected wavebands with $\mathrm{R} \sim 500-2000$. The spatial resolution is limited by the seeing disk which is typically $1-1.5^{\prime \prime}$. The dual output design of SpIOMM ensures that virtually every photon collected by the telescope reaches the detectors and is analyzed. We have however worked until now with only one detector, a Princeton Instrument CCD camera with an array of $1340 \times 130020 \mu$ m-pixels (corresponding to 0.55 arcsecond on the sky). A data cube thus results in 1.7 million spectra. Details on SpIOMM are presented by Drissen et al. (2010) and Bernier et al. (2008).

While the number of scientific applications of an imaging FTS is potentially very large, our group has focused on the interstellar medium of our own galaxy, the Milky way, and nearby spiral galaxies. Powerful constraints on models of galactic chemical evolution, on the star formation histories of galaxies and on the dynamical processes that transform them can be derived from accurate and homogeneous determinations of chemical 
abundances in individual gaseous nebulae, the distribution of their stellar populations in terms of age and metallicity, and the gaseous and stellar kinematics. SpIOMM is an ideal instrument to conduct a systematic study of abundances in nearby galaxies and thus easily detect evidence for small-scale enrichments and establish conditions under which they take place. The possibility to study the multiple emission line ratios and kinematics for hundreds of HII regions simultaneously in each individual galaxy is an excellent illustration of the niche for an imaging FTS. We have targeted a dozen galaxies so far (see Rousseau-Nepton et al., these proceedings, for two examples), and present in the next section some preliminary results on the nearby interacting spiral galaxy M51.

\section{Abundance gradients in M51}

The nearby interacting spiral galaxy M51 is a target of choice to study abundance gradients and localized enrichments. Although abundances of a small number of HII regions have been obtained with long slit spectrographs (Bresolin et al. 2004) and more recently integral field spectroscopy of the inner arcminute with VIRUS-P (Blanc et al. 2009), a global abundance study remains to be done. We have thus observed M51 with SpIOMM in two wavebands (450-510 nm and $650-680 \mathrm{~nm}$ ) and extracted line intensities for $\mathrm{H} \alpha, \mathrm{H} \beta$, [OIII] 495.9, 500.7, [NII] 654.8, 658.4, [SII] 671.7, 673.1. We have compared our emission line flux and line ratios with those of Blanc et al. (2009) and found an excellent agreement. We present in Fig. 1 the galactocentric variation in the $[\mathrm{NII}] / \mathrm{H} \alpha$ ratio from the core up to the outer region, where the northern arm of M51 overlaps with its companion, NGC 5195. This ratio is very large in the core of the galaxy, dominated by the AGN (see also Fig. 2). But we also notice a surprising global increase in the $[\mathrm{NII}] / \mathrm{H} \alpha$ ratio from the central region $(\sim 0.2)$ up to about $150^{\prime \prime}(\sim 0.4)$, followed by a flattening in the outer regions. Such a behavior is rather unusual, except maybe in the case of strongly interacting galaxies.

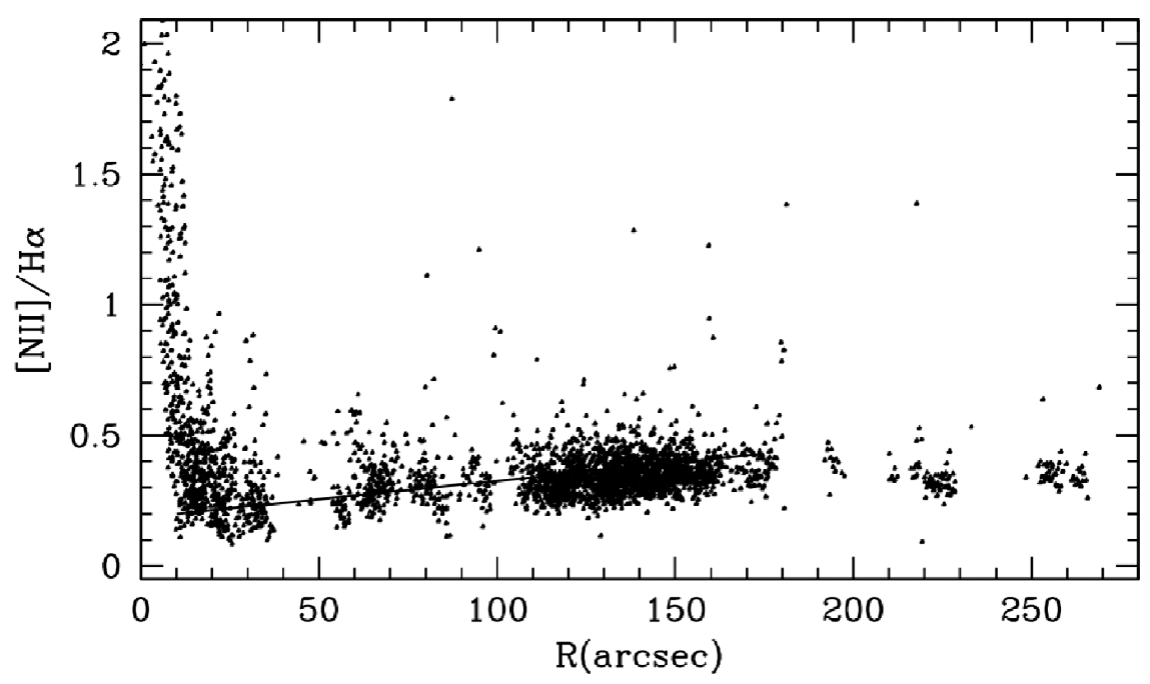

Figure 1. $\mathrm{H} \alpha /[\mathrm{NII}]$ ratio as a function of the galactocentric radius (no deprojection factor has been applied). Notice the high ratio in the core of the galaxy (due to the AGN) and the small increase with radius up to $\sim 170^{\prime \prime}$. 


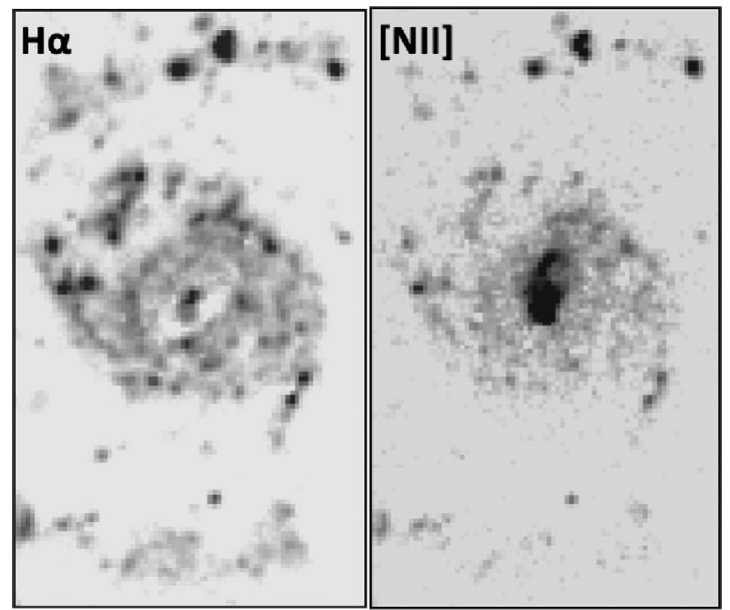

Figure 2. Central region of $\mathrm{M} 51$ in $\mathrm{H} \alpha$ and [NII]. The continuum has been subtracted. North is up, East to the left. Note the strong, extended [NII] region in the core due to the AGN.

\section{SITELLE}

We have recently secured funding to design and build an improved version of SpIOMM for the CFHT. SITELLE, another collaboration between Université Laval and ABBBomem, will have the same field of view, $12^{\prime} \times 12^{\prime}$, with $0.35^{\prime \prime}$ pixels (with two $2 \mathrm{k} \times 2 \mathrm{k}$, $15-\mu \mathrm{m}$ pixels CCDs as detectors), and will see its first light, if all goes according to plans, in early 2013. Because the CFHT has a larger primary mirror than the OMM telescope (3.6m vs. $1.6 \mathrm{~m}$ ) a better sky transparency (especially in the near UV; the CFHT is located at an altitude of $4200 \mathrm{~m}$ ) and better optics quality, we expect that SITELLE will offer a factor of 15 improvement over SpIOMM in terms of global efficiency. Because of its simple design (a simple focal reduced combined with a Michelson interferometer) and since it does not use slitlets, microlenses or fibers, SITELLE's field of view and global throughput will be significantly larger than that of other types of IFUs. As such, it will be an ideal instrument to analyze very faint targets (like the diffuse inter-arm ionized gas in galaxies or the intra-cluster medium), very faint diagnostic lines (such as [NII] 575.5 or [OIII]436.3 ) in extended objects. SITELLE will also be able to characterize the old stellar population with the detection of absorption lines such as FeII (527.0 and 533.5 $\mathrm{nm})$, the $\mathrm{H} \beta$ absorption or the Ca triplet $(849.8 \mathrm{~nm}, 854.2 \mathrm{~nm}$ and $866.2 \mathrm{~nm})$. These indices are then used to constrain the age of the population and the stellar metallicity.

\section{References}

Bacon, R., et al. 2001 MNRAS, 326, 23

Bacon, R., et al. 2010 SPIE, 7335, 7B

Bernier, A.-P., Charlebois, M., Drissen, L., \& Grandmont, F. 2008 SPIE, 7014, 70147J

Blanc, G. A., Heiderman, A., Gebhardt, K., Evans, N. J., \& Adams, J. 2009, ApJ, 704, 842

Bresolin, F., Garnett, D. R., \& Kennicutt, R. C. 2004 ApJ, 615, 228

Drissen, L., et al. 2008 SPIE, 7014, 70147K

Drissen, L., et al. 2011, in Fourier Transforms, Theory and Applications, Intech, in press

Flasar, F.M., et al. 2004 SSRv, 115, 169

Griffin, M. J., et al. 2010 A\&A, 518, L37

Maillard, J.-P., Drissen, L., Grandmont, F., \& Thibault, S. 2011 Exp. Astr., submitted

Panuzzo, P., et al. 2010 A\&A, 518, L3

Paumard, T., Maillard, J.-P. \& Morris, M. 2004 A $E A$, 426, 81 